\title{
Influence of medication risks and benefits on treatment preferences in older patients with multimorbidity
}

This article was published in the following Dove Press journal:

Patient Preference and Adherence

21 January 2017

Number of times this article has been viewed

\author{
Gillian E Caughey ${ }^{1,2}$ \\ Kirsty Tait ${ }^{3}$ \\ Agnes IVitry ${ }^{3}$ \\ Sepehr Shakib ${ }^{2,4}$ \\ 'Quality Use of Medicines and \\ Pharmacy Research Centre, Sansom \\ Institute for Health Research, \\ School of Pharmacy and Medical \\ Sciences, University of South \\ Australia, ${ }^{2}$ Department of Clinical \\ Pharmacology, Royal Adelaide \\ Hospital, ${ }^{3}$ School of Pharmacy and \\ Medical Sciences, University of South \\ Australia, ${ }^{4}$ Discipline of Pharmacology, \\ School of Medicine, University of \\ Adelaide, North Terrace, Adelaide, SA, \\ Australia
}

Abstract: Multimorbidity is associated with use of multiple medicines, increased risk of adverse events and treatment conflicts. This study aimed to examine how older patients with multimorbidity and clinicians balance the benefits and harms associated with a medication and in the presence of competing health outcomes. Interviews were conducted with 15 participants aged $\geq 65$ years with 2 or more chronic conditions. Three clinical scenarios were presented to understand patient preference to take a medicine according to i) degree of benefit, ii) type of adverse event and impact on daily living and iii) influence of comorbid conditions as competing health outcomes. Semi-structured interviews were also conducted with participants $(n=15)$ and clinicians $(n=5)$ to understand patient preferences and treatment decisions, in the setting of multimorbidity. The median age of participants was 79 years, $55 \%$ had 5 or more conditions and $47 \%$ took 8 or more medicines daily. When the level of benefit of the medicine ranged from $14 \%$ to $70 \%, 80 \%$ of participants chose to take the medicine, but when adverse effects were present, this was reduced to $0-33 \%$ depending upon impact on daily activities. In the presence of competing health outcomes, $13 \%-26 \%$ of patients chose to take the medicine. Two-thirds of patients reported that their doctor respects and considers their preferences and discussed medication benefits and harms. Interviews with clinicians showed that their overall approach to treatment decision-making for older individuals with multimorbidity was based upon 2 main factors, the patients' prognosis and their preferences. The degree of benefit gained was not the driver of patients' preference to take a medicine; rather, this decision was influenced by type and severity of adverse effects. Inclusion of patient preferences in the setting of risks and benefits of medicines with consideration and prioritization of competing health outcomes may result in improved health outcomes for people with multimorbidity.

Keywords: multimorbidity, comorbidity, patient preference, patient decision-making, medicines, geriatrics

\section{Introduction}

Multimorbidity is common in the older population, and over $60 \%$ of those aged 65 years and older will have 2 or more chronic conditions. ${ }^{1}$ Multimorbidity is associated with polypharmacy, high prevalence of treatment conflicts and potentially as a consequence, increased risk of medicine-related adverse events. ${ }^{2}$ Treatment conflicts arise when the treatment of one condition results in the worsening of another condition present in a patient, as a result of medicine--medicine or medicine-disease interactions. ${ }^{3-5}$ Further, the majority of current clinical guidelines fail to provide specific recommendations for treatment in the setting of multimorbidity. ${ }^{6,7}$ This can lead to complex and difficult management decisions for both the patients and the clinicians caring for them. ${ }^{8,9}$
Correspondence: Gillian E Caughey Quality Use of Medicines and Pharmacy Research Centre, Sansom Institute for Health Research, School of Pharmacy and Medical Sciences, University of South Australia, GPO Box 247I, Adelaide, SA 500I, Australia

Tel +6I 883021749

Email gillian.caughey@unisa.edu.au 
Use of medicines is associated with both benefits and harms, and how to balance these is particularly important in the setting of multimorbidity and associated use of multiple medicines. The treatment of those with multimorbidity commonly involves trade-offs in the setting of competing health priorities. ${ }^{8,10}$ As an example, in older patients with osteoarthritis (OA), one trade-off typically encountered is that they may wish to use a nonsteroidal anti-inflammatory drug (NSAID) to relieve their symptoms, at the expense of controlling their hypertension or risk of gastrointestinal adverse events. Clinicians and patients may consider different outcomes when making health-related decisions. Tension between therapeutic goals of patients and their health care providers is common; in 30\% of occasions, the patients' main priority for their health was not in the top 3 of their health care providers. ${ }^{11}$ This discordance was greatest in those with multimorbidity or competing demands. Presenting and incorporating competing priorities in the decision-making process in terms of global health outcomes including survival, quality of life, symptom relief and current physical and cognitive function, rather than disease-specific outcomes, is particularly important for patients with multimorbidity. ${ }^{12}$

Adverse events associated with medicine use, even those considered "less severe or side effects" are an important determinant of a patient's willingness to commence or continue with a medication. ${ }^{8,13}$ A recent study found that older persons' willingness to take a medication for cardiovascular disease (CVD) was more related to potential adverse events than to potential benefits. ${ }^{13}$ The incorporation of patients' preferences for treatment into clinical decision-making is essential to patient-centered care ${ }^{14}$ and is particularly pertinent in the context of multimorbidity and the presence of competing health outcomes. This study aimed to examine how older patients with multimorbidity balance the benefits and harms associated with a medication, and in the presence of competing health outcomes. In addition, the factors that clinicians consider when making treatment decisions for this particular patient group were examined.

\section{Methods}

\section{Study sample}

Participants were recruited from 2 Multidisciplinary Ambulatory Consulting Service (MACS) clinics at tertiary teaching hospitals from June 2012 to September 2012. The MACS clinics are a holistic management model for older patients with multiple comorbidities recently discharged from hospital and subsequently referred to the MACS clinic for outpatient follow-up. The MACS model of care is based on a multidisciplinary health care team and assessment, with determination of individualized agreed evidence-based goals. ${ }^{15}$ It has been shown to be associated with high compliance to clinical guideline recommendations for all comorbid conditions present, including both non-pharmacological and pharmacological recommendations and health service utilization. ${ }^{15}$ Included in the study were those patients aged 65 years or older with English proficiency and 2 or more chronic conditions. Patients were excluded from the study if they had a formal diagnosis of dementia. The Human Research Ethics Committees of Royal Adelaide Hospital and University of South Australia approved this study. All participants included in the study provided written informed consent.

\section{Study design and data collection}

Participants underwent face-to-face interviews, which were conducted by a clinical pharmacist (KT) at the recruitment sites. Sociodemographic variables included gender, age, race, marital status, education level and living situation. Clinical variables including current medications and chronic conditions were determined from a brief medical history in addition to patient records (including preadmission questionnaires and clinicians' referral letters to general practitioners [GPs]). To assess self-rated health, participants were asked, "In general how would you rate your present health?", with 5 response categories (poor, fair, good, very good or excellent). For the analyses of specific disease combinations, the responses were grouped into 2 categories: i) poor or fair and ii) good, very good or excellent. Functional status was assessed using instrumental activities of daily living (ADLs), determined using the Barthel index, with a range from 0 (dependent) to 100 (independent). ${ }^{16}$

Using similar methodology to a previous study from the US, we examined the use of medication for prevention of CVD (namely myocardial infarction [MI]) to understand patient preferences in our study. ${ }^{13}$ CVD is highly prevalent in the older Australian population and contributes to more than $50 \%$ of the overall burden of disease in the older population. ${ }^{17}$ The primary outcome for the study was participants' preference to take a medication to prevent MI when differing levels of benefits and risks of medications were presented. The benefits of the medication were presented to participants in terms of a patient's baseline risk of MI, with and without the medication. In each scenario, patients were asked whether they would take the medication with the following response categories: "yes", "no" and "not sure". Scenarios were communicated to the patients verbally and through pictographs 
to facilitate understanding of the benefit/risk information presented. ${ }^{18}$

In the first series of questions, the patients' baseline risk of MI as well as the relative risk reduction (RRR) with medication varied to determine the influence of varying levels of benefit on patients' willingness to take the medication. To understand the influence of benefit alone on patient preference to take the medication, in this set of scenarios, the medication was said to be free of adverse effects. The level of baseline risk of MI varied as did the level of benefit associated with taking the medicine. The initial baseline risk of MI was 1 in $5(20 \%)$ which was derived from the Framingham Heart Study ${ }^{19}$ as previously described. ${ }^{13}$ Consistent with the average absolute risk reduction found in clinical trials assessing the use of antihypertensives and statins for primary prevention of CVD, ${ }^{20,21}$ pharmacological intervention was said to provide a 30\% RRR, decreasing the 5-year risk of MI to 14 in 100 $(14 \%) .{ }^{13}$ The baseline risk of MI ranged from 1 in $10(10 \%)$ to 4 in $10(40 \%)$, and the risk of MI with the medication ranged from $10 \%$ to $70 \%$.

In order to assess the influence of adverse events on patient preference to take a medication, patients were presented with scenarios in which medication use was associated with differing adverse effects that either did not or did impact on daily activities. ${ }^{13}$ Adverse effects included daily fatigue and weakness, daily nausea or daily dizziness and headache, and were identified as common adverse effects associated with cardiovascular medications from the Australian Medicines Handbook. ${ }^{22}$ The baseline risk in these scenarios as well as the RRR with medication remained constant; the baseline MI risk was $20 \%$, and the medication reduced the risk to $14 \%$.

We also examined the influence of competing health outcomes on patient preferences for treatment. OA and chronic respiratory disease are common comorbid conditions in older patients with CVD, which are associated with treatment conflicts and are competing health priorities. ${ }^{4}$ The use of NSAIDs and corticosteroids for OA and respiratory disease, respectively, is detrimental to cardiovascular health, with reports of approximately a 10\% increase in risk of MI being associated with the use of NSAIDs or corticosteroids in the older population. ${ }^{23,24}$ Participants presented with a baseline risk of MI of 1 in 5 (20\%) and were asked if they would be willing to take a medication to relieve joint pain for OA, if it increased their risk of MI to 3 in $10(30 \%)$. Similarly for respiratory disease, participants presented with a baseline risk of MI of 1 in $5(20 \%)$ and were asked if they would be willing to take a medication to improve breathing, if it increased their risk of MI to 3 in $10(30 \%)$.
Qualitative interviews examining patient and clinician views of treatment decisions for older patients with multimorbidity

Upon conclusion of the benefit-and-harm scenario interview, patients were next asked a series of semi-structured interview questions regarding i) treatment preferences, ii) discussion of medication adverse effects and iii) shared decision-making. The interview guide included open-ended questions based on these 3 key topics of interest.

To understand clinician's views on treatment decisions, semi-structured open-ended interview, with questions focused on 3 key areas of interest which included i) their overall approach to treatment decision-making for older patients with multimorbidity, ii) patient preferences and iii) polypharmacy, was conducted by the same clinical pharmacist (KT) who performed the patient interviews. The interview also assessed the influence of patients' prognosis and their preferences on their decision-making.

The interviews were recorded in digital audio format, and notes taken throughout the interview to document nonverbal communication and context.

\section{Data analysis}

Descriptive statistics (proportions, means and standard deviations [SDs]) were used to describe the study cohort's sociodemographics and clinical characteristics and patient preferences to take medication, under the various scenarios presented. For the qualitative studies, the content of each interview was transcribed verbatim, and the transcripts were then thematically analyzed by 2 independent researchers (GEC and KT) to identify key common emerging themes using a constant comparison approach. ${ }^{25}$

\section{Results}

A total of 15 patients and 5 clinicians completed the study interviews. The patient demographics and clinical characteristics are presented in Table 1. Overall, the presence of multiple chronic conditions and medicine use were common with an average of $6( \pm \mathrm{SD} 4.3)$ chronic conditions and 8 ( \pm SD 3.7$)$ regular prescription medications. All patients were taking at least 1 cardiovascular medicine.

Examination of the effect of the degree of benefit showed that use of a once-daily medication that was free of adverse effects, which provided a $30 \%$ RRR to the baseline risk of MI (an absolute risk reduction of 6 fewer individuals experiencing an MI), resulted in the majority of patients $(80 \%)$ indicating that they would take the medication (Figure 1). In scenario 2, the absolute benefit of the medication increased, 
Table I Demographics and clinical characteristics of study cohort $(n=15)$

\begin{tabular}{|c|c|}
\hline Patient characteristics & $\begin{array}{l}\text { Number (\%) (unless } \\
\text { otherwise stated) }\end{array}$ \\
\hline \multicolumn{2}{|l|}{ Demographics } \\
\hline Gender, male & 7 (47\%) \\
\hline Age, median (IQR) & $79(73-86)$ \\
\hline \multicolumn{2}{|l|}{ Education level } \\
\hline Primary & $10(67 \%)$ \\
\hline Secondary & $3(20 \%)$ \\
\hline Tertiary & $2(13 \%)$ \\
\hline \multicolumn{2}{|l|}{ Marital status } \\
\hline Single & $2(13 \%)$ \\
\hline Married & II (73\%) \\
\hline Divorced & I (7\%) \\
\hline Widowed & $\mathrm{I}(7 \%)$ \\
\hline Independent living (\% yes) & $15(100 \%)$ \\
\hline \multicolumn{2}{|l|}{ Clinical characteristics } \\
\hline \multicolumn{2}{|l|}{ Self-rated health } \\
\hline Excellent & $0(0 \%)$ \\
\hline Very good & $3(20 \%)$ \\
\hline Good & $2(13.5 \%)$ \\
\hline Fair & $8(53 \%)$ \\
\hline Poor & $2(13.5 \%)$ \\
\hline \multicolumn{2}{|l|}{ Functional status (ADLs, Barthel index score) } \\
\hline 0-25 (difficulty with ADLs) & $0(0 \%)$ \\
\hline $25-50$ & $2(13 \%)$ \\
\hline $50-75$ & $0(0 \%)$ \\
\hline 75-100 (limited/no difficulty with ADLs) & $13(87 \%)$ \\
\hline \multicolumn{2}{|l|}{ Number of chronic conditions } \\
\hline $2-4$ & $7(47 \%)$ \\
\hline $5-7$ & $5(33 \%)$ \\
\hline$\geq 8$ & $3(20 \%)$ \\
\hline Average number of chronic conditions $( \pm S D)$ & $6( \pm 4.26)$ \\
\hline \multicolumn{2}{|l|}{ Number of regular prescription medications } \\
\hline $0-4$ & $2(13 \%)$ \\
\hline $5-7$ & $6(40 \%)$ \\
\hline$\geq 8$ & $7(47 \%)$ \\
\hline Average number of regular prescriptions $( \pm S D)$ & $8( \pm 3.7)$ \\
\hline
\end{tabular}

Abbreviations: IQR, interquartile range; ADLs, activities of daily living; SD, standard deviation.

a $50 \%$ RRR of MI was presented and the proportion of patients willing to take the medication decreased. Overall, $67 \%$ responded that they would take the medication, but $26 \%$ of patients presented with this scenario were uncertain (Figure 1). In scenario 3 where the baseline risk of MI was $40 \%, 80 \%$ of patients indicated that they would be willing to take a medication free of adverse effects which would provide a 75\% RRR of MI (Figure 1). In the final scenario presented where the baseline risk of MI with the medication was only $10 \%, 80 \%$ of patients said they would take the particular medication even if it provided a $30 \%$ decrease in relative risk. For all scenarios presented, there was little change in the number of patients unwilling to take the medication.
Examination of the effect of harm on patients' willingness to take the medication showed that over $50 \%$ of the patients examined were not willing to take a medication if it had an equivalent degree of benefit and risk (Figure 2). One-half reported that they would not take or were unsure whether to take a medication if it was associated with mild fatigue and weakness that would not impact upon performing ADLs. The same result was seen when patients were presented with a medication associated with dizziness and headache, but mild enough not to impact upon ADL. A larger proportion (67\%) were unwilling to take or unsure about taking a medication with mild nausea as an adverse effect, even if it did not impact on ADL (Figure 2).

Of the participants willing to tolerate these mild adverse effects, less than a third remained willing if the adverse effects were severe enough to interfere with ADL. Fatigue and weakness, impacting on ADL, was the least severe of the adverse events in this regard, with 33\% reporting a willingness to take a medication with this harm profile. Only $17 \%$ of patients were willing to take a medication that was associated with dizziness and headache, impacting on ADL. By contrast, none of the patients were willing to take a medication associated with mild nausea that impacts on ADL (Figure 2).

We next examined patient preferences in the context of competing health outcomes for use of medications to alleviate symptoms associated with common comorbid conditions, OA or chronic respiratory disease. In our study cohort, $66.7 \%$ of the patients had OA, and $26.7 \%$ had chronic respiratory disease. For OA, $80 \%$ of patients would not take a medication to relieve joint pain if it was associated with a $10 \%$ increased risk of MI. By contrast, $54 \%$ of patients would not take a medication to improve breathing if it would increase their risk of MI by $10 \%$, with a greater proportion of uncertainty in this context, with $20 \%$ unsure of what to prioritize (Figure 3).

\section{Qualitative studies focused on patient and clinician preferences}

\section{Patient interviews}

When patients were asked about their perspectives on individual patient preferences and shared decision-making, $60 \%$ $(n=9)$ said they regularly discussed their treatment preferences with their doctor. Common topics that participants said they discussed with their clinicians included rationale for treatment, impact of medication on functioning, ability of the medication to provide symptomatic relief and the use of alternative therapies. However, of these, $22 \%(n=2)$ stated 


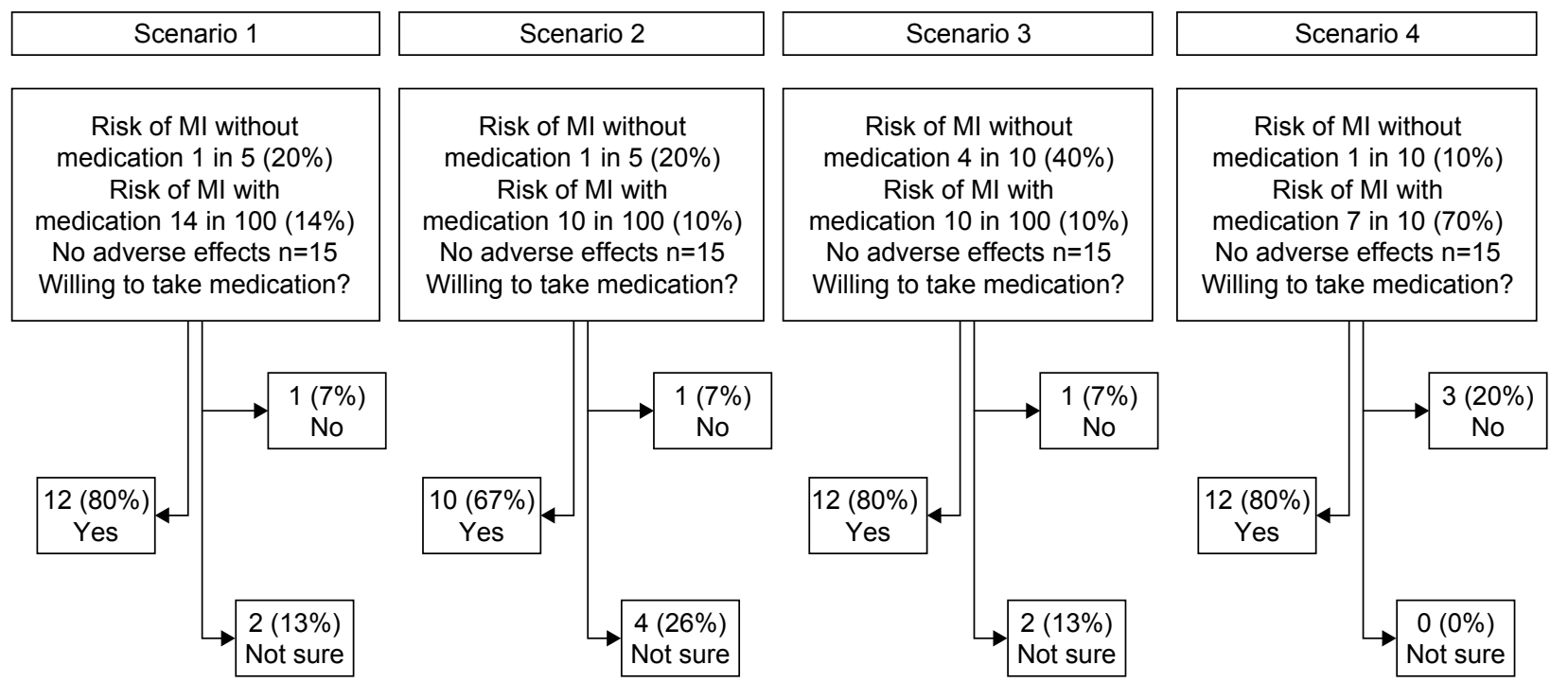

Figure I Patient preference to take medication for primary prevention of cardiovascular disease according to degree of benefit only. Abbreviation: Ml, myocardial infarction.

they would ultimately agree to follow the doctor's preferred approach for treatment, and $22 \%$ also stated that they thought their doctors did not encourage such discussions. The reasons provided by those who did not discuss their treatment preferences with their doctor included the following: the doctor is the expert $(\mathrm{n}=3)$ and I trust my doctor $(\mathrm{n}=2)$. Overall, almost three-quarters of the patients $(\mathrm{n}=11)$ felt their doctor respects and considers their preferences.

The majority of patients $(66 \%, n=10)$ recalled that their doctor discussed the benefits and risks associated with

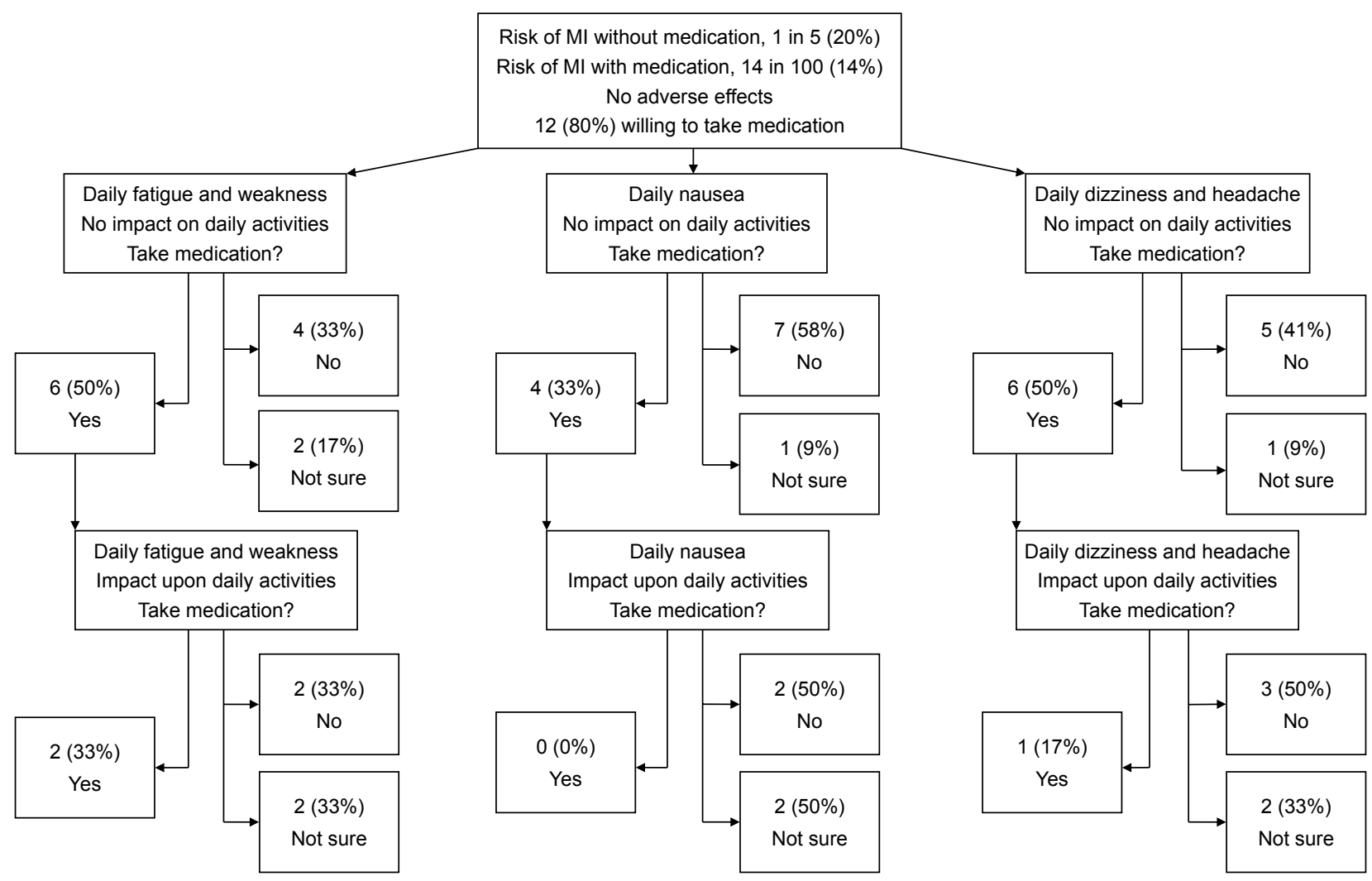

Figure 2 Patient preference to take medication for primary prevention of cardiovascular disease according to type of adverse event and impact on daily activities. Abbreviation: MI, myocardial infarction. 

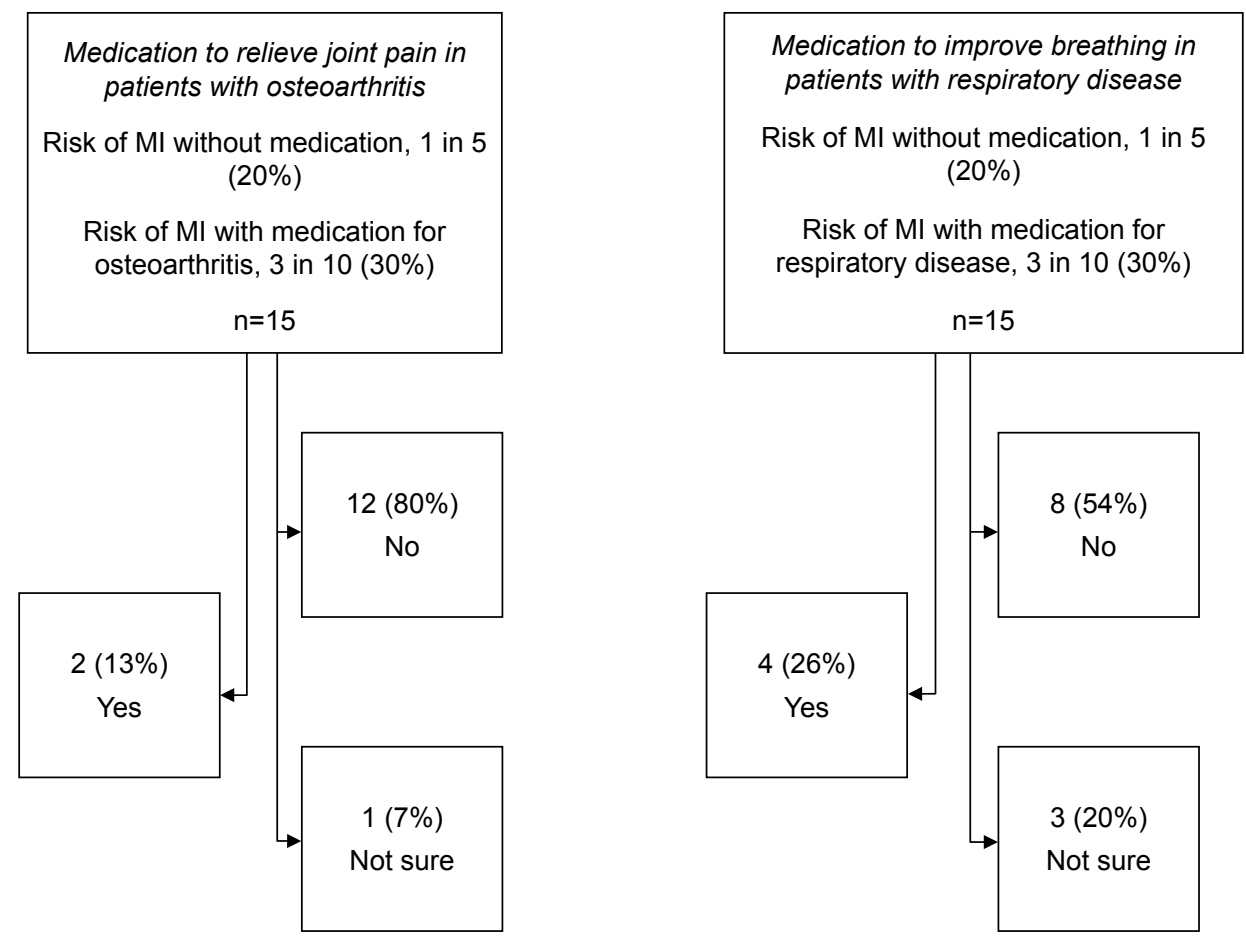

Figure 3 Patient preference to take medication for common comorbid conditions, osteoarthritis or respiratory disease in the context of competing health outcomes. Abbreviation: MI, myocardial infarction.

medications. For the remaining 5 patients, discussions were limited to adverse effects of medications only or benefits of medications only or no discussion on either the benefits or the risks of medications took place.

When questioned about shared decision-making, overall, the patient cohort did not desire to have greater participation in making health care decisions, with 10 of the 15 patients stating they feel their preferences and opinions are heard and feel content with their current physician-patient dynamic. The 5 patients who wished to be more involved felt that it would be unrealistic to expect greater participation in the complex setting of their care. Consideration of patients' values and beliefs was also found to be important. Patients stated they would like to be more informed about their condition(s) and the various treatment options available.

\section{Clinician interviews}

Interviews with clinicians showed that their overall approach to treatment decision-making for older individuals with multimorbidity was based upon 2 main factors: the patients' prognosis and their preferences. These preferences included either improving quality of life by relieving patient's symptoms, or decreasing mortality via the use of medications to prolong life. Clinicians talked about adapting treatment depending upon the patient's symptoms and not always treating to therapeutic targets. Some clinicians mentioned prioritizing conditions that were causing the most trouble to their patient. The aspect of patient preference was also recognized as extremely important due to competing interests. Clinicians commented that the treatment of younger individuals with a single chronic condition is approached in a very similar way, reiterating the importance of patient preference, but that they can be treated more aggressively. On the whole, in younger patients, their general aim was to improve mortality as opposed to providing short-term, symptomatic relief. Treating younger patients was said to be less complex due to the absence of competing health outcomes, general use of fewer medications and decreased potential for drug interactions.

Clinicians spoke of numerous difficulties when applying treatment guidelines for older patients with multiple chronic diseases. All mentioned the poor applicability of current guidelines to their patients, with several participants noting that current guidelines are largely based upon data from clinical trials involving younger patients with a single-disease state, which is not the reality of most patients that they see. As a result, clinicians reported having to juggle several treatment guidelines when treating multiple conditions. This can lead to discrepancies between treatment targets for 
different conditions, treatment conflicts and polypharmacy. Furthermore, physicians appeared frustrated with the guidelines because they tend to recommend medications that will provide long-term benefits, which was often deemed to be of lesser importance for older individuals given their increased age and different treatment goals. Again, consideration of patient preference and goals for treatment was regarded as fundamental to therapeutic guideline applicability. Several other factors were said to influence the utilization of clinical practice guidelines, including quality of life, social supports, functional status, adverse effects and medication adherence and accessibility.

Competing health outcomes and polypharmacy were common issues faced by the clinicians in this study. When dealing with balancing the competing health interests, clinicians reported that they tend to base their decisions upon the current stage of the disease and its likely progression, while incorporating the preferences of the patient. Polypharmacy was not viewed as negative when caring for older people with multiple conditions as long as it resulted in an overall net benefit, with regular medication review and rationalization of the treatment regimen. If the medications are causing harm, or little or no benefit, then clinicians commented that they would suggest the patients to stop or decrease some medications. A number of clinicians also mentioned about deprescribing; however, some reported difficulties in taking this approach. For example 1 participant said that medications are often restarted by GPs in the community. Another reported they commonly found patients to be uncomfortable when they were suggested cessation of medication(s). The importance of allowing the patient to take control of the deprescribing process, to alleviate any anxiety to him/ her, was also highlighted. One physician said that Home Medicine Reviews are of great benefit in resolving the issue of polypharmacy but expressed frustration in regard to their limited accessibility.

Education and communication were the key factors identified to reduce the likelihood of adverse effects associated with polypharmacy and complex drug regimens. Clinicians spoke of the importance of educating the patients and their family/carer, and timely and efficient communication between health care providers, namely the GP and pharmacist. Apart from minimizing the number of prescribed medications, other strategies included asking patients about previous adverse drug events, checking medication interactions prior to prescribing, avoiding complex and predictable drug interactions and regularly reviewing drug regimens.

\section{Discussion}

In the current study of older patients with multimorbidity, the degree of benefit gained from a medicine was not the driver of patients' preference to take the medication. Rather, this decision was largely influenced by the type and severity of adverse effects associated with the medicine. This was further confirmed in the presence of competing health priorities where the use of a medicine for 1 condition may result in increased risk of harm associated with another condition. We found that the type of competing priority influenced therapeutic decisions of patients, illustrating the impact of the type of comorbid disease upon patients' willingness to take a medication. Patients were less likely to take a medicine to obtain symptomatic relief of joint pain if it increased their risk of MI, but were more likely to take a medication to improve breathing in respiratory disease with the same risk of MI. These results highlight the importance of including patient preferences for treatment in the setting of risks and benefits of medicines for all conditions present in an individual.

The findings from this Australian study are concordant with previous findings where adverse effects of a medicine had the greatest influence on patient preferences to take a medicine. ${ }^{13,26}$ Patients' willingness to take medication was relatively insensitive to the benefits associated with therapy. In a similar study of 356 patients in the US, where the benefits and risks associated with medications for primary CVD prevention were examined as in the current study, between $48 \%$ and $69 \%$ of participants were unwilling to take a medicine if it was associated with mild fatigue, nausea or fuzzy thinking. If the medication caused adverse effects that affected functioning, only $3 \%$ would take the medication. This is in contrast to $88 \%$ of people who responded they would take the medicine if it were free of adverse effects. ${ }^{13}$ In another study of patients' preferences regarding the treatment of knee $\mathrm{OA}$, the risk of common medication adverse effects including gastrointestinal ulcer had the strongest impact upon patients' choice to take a medicine. ${ }^{26}$

These findings have numerous implications for the development and utilization of clinical treatment guidelines for multimorbid patients. Firstly, guideline developers must recognize that medication adverse effects are generally considered as a competing outcome, independent of their severity. ${ }^{13}$ This is particularly pertinent for older individuals with multimorbidity given their use of multiple medications, which predisposes them to an increased risk of experiencing an adverse event. ${ }^{2}$ Another important consideration is the type of adverse event and the patient's previous experiences with medications and adverse events, as shown in 
the current study. The decision to start a medication should be individualized and not solely based upon the benefits of the intervention alone. Inclusion of individual patient preferences where clinicians are able to tailor their treatment approach to suit the preferences and priorities of their patient, while aiming to fulfill their overall therapeutic goal, is the key to the provision of patient-centered care. ${ }^{2,27} \mathrm{Com}-$ munication of risks and benefits of medications, within the context of multimorbidity, is an important component, and as this study shows, decision-making for patients is highly dependent on adverse events, rather than health benefits. Compliance with medication regimens for patients with chronic diseases is crucial to achieve treatment success and is a key challenge for health professionals worldwide. ${ }^{28}$ Adverse events and a lack of patient engagement in the decision-making process have been identified as contributors toward noncompliance. ${ }^{29,30}$ A better understanding of the factors around patients' willingness to take medications will ultimately lead to improved compliance, and may help to identify those most at risk of noncompliance and ultimately poorer health outcomes.

A number of our findings are consistent with previous studies, in regard to decision-making around health care. Prior research has concluded that there is significant variation among patients' willingness to participate, and many patients do not wish to actively participate in prescribing decisions. Of those willing to participate, the spectrum of involvement ranges from requesting information about alternative treatments to enquiring about medication adverse effects. ${ }^{2,27}$ In contrast to previous studies, we found that a large proportion of patients reported that their clinician regularly informed them about the benefits and risks of therapy prior to treatment initiation. Previous studies have reported that physicians do not routinely discuss the benefits and risks of medicines, due to time constraints and unrealistic patient expectations..$^{9,31,32}$ It is likely that the provision of multidisciplinary collaborative team-based care that includes patient preferences and patient goals within the MACS model of care facilitated the discussion of medication risks and benefits in this setting. Most patients were content with their relationship towards their prescriber and did not desire to have a greater influence upon the outcome of their health. This theme is common among similar studies, with patients' trust in their doctor and their lack of medication knowledge being barriers to patient participation. ${ }^{27}$ From the results obtained, it appears that patients who actively contribute to the decision-making process may also require additional information regarding their therapeutic options. However, despite wishing to receive additional information, the majority of patients do not want to be involved in health care decisions to a greater degree.

The perspective of clinicians regarding overall approach to treatment decision-making for older patients with multimorbidity highlights the complexity of caring for these patients, in particular the uncertainty of applying diseasespecific guidelines that may not be relevant for older patients with multimorbidity or in alignment with patient preferences and priorities. While the importance of individual patient's prognosis and treatment goals was deemed fundamental in determining the course of treatment for older patients with multimorbidity, there was variability in how to best incorporate this in the decision-making process. While not observed in our study, conflict between clinicians and patient goals commonly occurs, and it has been suggested that clinicians would benefit from tools to specifically assist in shared decision-making, which include approaches to reconciling their own and their patients' priorities. ${ }^{9}$ Outcomes data on treatment strategies in this population and alternative guidelines will help to improve decision-making. ${ }^{9}$ The US clinicians have also highlighted the importance of assessing a patient's function, support networks and evidence of prior non-adherence to alleviate issues of polypharmacy and complex drug regimens. ${ }^{9}$

This study had several limitations. The sample size was small and reflective of the multimorbid and complex care needs of the participants and their current overall health status as fair or poor and may not be reflective of the majority of older Australians. The general older Australian population rate their health as good or above, ${ }^{33}$ potentially limiting the applicability of the findings. Further, we have previously shown that the MACS clinic is associated with higher compliance to clinical guideline recommendations for all comorbid conditions present, including both non-pharmacological and pharmacological recommendations and health service utilization, ${ }^{15}$ and this may also limit applicability to the older multimorbid population. While the study was conducted at 2 sites, the treating clinicians were the same at both the sites. The study questionnaire focused on patients' willingness to take medication for primary prevention of CVD, and how the results obtained relate to other health conditions is unclear. The benefit of the medication was presented in terms of reduction in the risk of $\mathrm{MI}$, as opposed to the effect on universal health outcomes, such as mortality risk, symptom relief or functional ability, which may be more applicable to those with multimorbidity. ${ }^{13}$ However, a recent study 
reported that the use of universal health outcomes had limited applicability when determining patients' priority for taking a medication. ${ }^{34}$

\section{Conclusion}

This study shows that patients' willingness to take medication is not directly influenced by the degree of benefit gained from pharmacological intervention. However, the type and severity of the adverse effect does significantly influence patients' willingness to take a medication. An increased understanding of the treatment decision-making process with consideration and prioritization of competing outcomes may result in improved health outcomes for individuals with multimorbidity.

\section{Acknowledgments}

The authors would like to thank Allyson Rappensberg and Georgina Walker for helping with patient recruitment. This work was supported by an Australian Government National Health and Medical Research Council Centre of Research Excellence in Post-Marketing Surveillance of Medicines and Medical Devices grant (GNT1040938). The funder had no role in the study design, collection, analysis and interpretation of data, writing of article or decision to publish.

\section{Disclosure}

The authors report no conflicts of interest in this work.

\section{References}

1. Caughey GE, Ramsay EN, Vitry AI, et al. Comorbid chronic diseases, discordant impact on mortality in older people: a 14-year longitudinal population study. J Epidemiol Community Health. 2010;64(12): 1036-1042.

2. Roughead EE, Vitry AI, Caughey GE, Gilbert AL. Multimorbidity, care complexity and prescribing for the elderly. Aging Health. 2011; 7(5):695-705

3. Caughey GE, Roughead EE, Shakib S, McDermott RA, Vitry AI, Gilbert AL. Comorbidity of chronic disease and potential treatment conflicts in older people dispensed antidepressants. Age Ageing. 2010;39(4): 488-494.

4. Caughey GE, Roughead EE, Shakib S, Vitry AI, Gilbert AL. Comorbidity and potential treatment conflicts in elderly heart failure patients: a retrospective, cross-sectional study of administrative claims data. Drugs Aging. 2011;28(7):575-581.

5. Caughey GE, Roughead EE, Vitry AI, McDermott RA, Shakib S, Gilbert AL. Comorbidity in the elderly with diabetes: identification of areas of potential treatment conflicts. Diabetes Res Clin Pract. 2010;87(3): 385-393.

6. Vitry AI, Zhang Y. Quality of Australian clinical guidelines and relevance to the care of older people with multiple comorbid conditions. Med J Aust. 2008;189(7):360-365.

7. Boyd CM, Darer J, Boult C, Fried LP, Boult L, Wu AW. Clinical practice guidelines and quality of care for older patients with multiple comorbid diseases: implications for pay for performance. JAMA. 2005; 294(6):716-724.

8. Fried TR, McGraw S, Agostini JV, Tinetti ME. Views of older persons with multiple morbidities on competing outcomes and clinical decisionmaking. J Am Geriatr Soc. 2008;56(10):1839-1844.
9. Fried TR, Tinetti ME, Iannone L. Primary care clinicians' experiences with treatment decision making for older persons with multiple conditions. Arch Intern Med. 2011;171(1):75-80.

10. Tinetti ME, McAvay GJ, Fried TR, et al. Health outcome priorities among competing cardiovascular, fall injury, and medication-related symptom outcomes. J Am Geriatr Soc. 2008;56(8):1409-1416.

11. Zulman DM, Kerr EA, Hofer TP, Heisler M, Zikmund-Fisher BJ. Patient-provider concordance in the prioritization of health conditions among hypertensive diabetes patients. J Gen Intern Med. 2010; 25(5):408-414.

12. Tinetti ME, Bogardus ST Jr, Agostini JV. Potential pitfalls of diseasespecific guidelines for patients with multiple conditions. New Engl J Med. 2004;351(27):2870-2874.

13. Fried TR, Tinetti ME, Towle V, O'Leary JR, Iannone L. Effects of benefits and harms on older persons' willingness to take medication for primary cardiovascular prevention. Arch Intern Med. 2011;171(10): 923-928.

14. Barry MJ, Edgman-Levitan S. Shared decision making - the pinnacle of patient-centered care. New Engl J Med. 2012;366(9):780-781.

15. Ho TH, Caughey GE, Shakib S. Guideline compliance in chronic heart failure patients with multiple comorbid diseases: evaluation of an individualised multidisciplinary model of care. PLoS One. 2014;9(4):e93129.

16. Sainsbury A, Seebass G, Bansal A, Young JB. Reliability of the Barthel Index when used with older people. Age Ageing. 2005;34(3):228-232.

17. Australian Institute of Health and Welfare. Cardiovascular disease series no. 35. Cat. no. CVD 53. Canberra: AIHW; 2011. Available from: http://www.aihw.gov.au/publication-detail/?id=10737418510. Accessed September 30, 2014.

18. Woloshin S, Schwartz LM. Communicating data about the benefits and harms of treatment: a randomized trial. Ann Intern Med. 2011; 155(2):87-96.

19. Wilson PW, D'Agostino RB, Levy D, Belanger AM, Silbershatz H, Kannel WB. Prediction of coronary heart disease using risk factor categories. Circulation. 1998;97(18):1837-1847.

20. Thavendiranathan P, Bagai A, Brookhart MA, Choudhry NK. Primary prevention of cardiovascular diseases with statin therapy: a metaanalysis of randomized controlled trials. Arch Intern Med. 2006;166(21): 2307-2313.

21. Staessen JA, Wang JG, Thijs L. Cardiovascular protection and blood pressure reduction: a meta-analysis. Lancet. 2001;358(9290): $1305-1315$.

22. Australian Medicines Handbook Pty. Ltd. Australian Medicines Handbook. Adelaide: Australian Medicines Handbook Pty. Ltd.; 2014.

23. Varas-Lorenzo C, Rodriguez LA, Maguire A, Castellsague J, PerezGutthann S. Use of oral corticosteroids and the risk of acute myocardial infarction. Atherosclerosis. 2007;192(2):376-383.

24. Pratt N, Roughead EE, Ryan P, Gilbert AL. Differential impact of NSAIDs on rate of adverse events that require hospitalization in highrisk and general veteran populations: a retrospective cohort study. Drugs Aging. 2010;27(1):63-71.

25. Boeije H. A purposeful approach to the constant comparative method in the analysis of qualitative interviews. Qual Quant. 2002;36(4): 391-409.

26. Fraenkel L, Bogardus ST Jr, Concato J, Wittink DR. Treatment options in knee osteoarthritis: the patient's perspective. Arch Intern Med. 2004; 164(12):1299-1304

27. Belcher VN, Fried TR, Agostini JV, Tinetti ME. Views of older adults on patient participation in medication-related decision making. J Gen Intern Med. 2006;21(4):298-303.

28. World Health Organisation. Adherence to Long Term Therapies: Evidence for Action. Geneva: World Health Organisation; 2003.

29. Epstein RM, Peters E. Beyond information: exploring patients' preferences. JAMA. 2009;302(2):195-197.

30. Burgess CL, Holman CD, Satti AG. Adverse drug reactions in older Australians, 1981-2002. Med J Aust. 2005;182(6):267-270.

31. Gilbert AL, Caughey GE, Vitry AI, et al. Ageing well: improving the management of patients with multiple chronic health problems. Australas J Ageing. 2011;30 Suppl 2:32-37. 
32. Stevenson FA, Barry CA, Britten N, Barber N, Bradley CP. Doctorpatient communication about drugs: the evidence for shared decision making. Soc Sci Med. 2000;50(6):829-840.

33. Australian Institute of Health and Welfare. Older Australia at a glance: 4th edition. Cat. no. AGE 52. Canberra: AIHW; 2007. Available from: http://www.aihw.gov.au/publication-detail/?id=6442468045. Accessed November 9, 2015.
34. Case SM, O'Leary J, Kim N, Tinetti ME, Fried TR. The relationship between universal health outcome priorities and willingness to take medication for primary prevention of myocardial infarction. $J \mathrm{Am}$ Geriatr Soc. 2014;62(9):1753-1758.

\section{Publish your work in this journal}

Patient Preference and Adherence is an international, peer-reviewed, open access journal that focuses on the growing importance of patient preference and adherence throughout the therapeutic continuum. Patient satisfaction, acceptability, quality of life, compliance, persistence and their role in developing new therapeutic modalities and compounds to optimize clinical outcomes for existing disease states are major areas of interest for the journal. This journal has been accepted for indexing on PubMed Central. The manuscript management system is completely online and includes a very quick and fair peer-review system, which is all easy to use. Visit http://www. dovepress.com/testimonials.php to read real quotes from published authors.

Submit your manuscript here: http://www.dovepress.com/patient-preference-and-adherence-journal 